\title{
Evaluation of Body Sway in a Seated Posture after Alcohol Ingestion with an Aim to Evaluate Motion Sickness Caused by Three-Dimensional Images
}

\section{Yasuyuki Matsuura ${ }^{1}$, Masaki Amemori², Tomoki Shiomi ${ }^{3}$, Masaru Miyao ${ }^{3}$, Kazuki Yoshikawa $^{4}$, Takayuki Hirata ${ }^{4}$ and Hiroki Takada ${ }^{4}$}

${ }^{1}$ Headquarters for Innovative Society-Academia Cooperation, University of Fukui 3-9-1, Bunkyo, Fukui, Japan

${ }^{2}$ Department of Human \& Artificial Intelligent System, Faculty of Engineering, University of Fukui, Japan

${ }^{3}$ Department of Information Engineering, Graduate School of Information Science, Nagoya University, Japan

${ }^{4}$ Department of Human \& Artificial Intelligent System, Graduate School of Engineering, University of Fukui, Japan

\begin{abstract}
A few studies have evaluated swaying motion of the body in the seated posture; however, these studies did not evaluate motion sickness caused by watching movies, traveling, etc. This study aimed to analyze stabilograms recorded while the individuals were seated. Stabilometry was performed on 7 healthy male subjects (age, 21-22 years) in the seated position $5 \mathrm{~min}$ before, and at 5-min intervals from 5 to 60 min after alcohol load. Alcohol caused a decrease in the cerebellar equilibrium function that controls the vestibulospinal reflex. We adjusted the degree of simulative effect on the human equilibrium function. The stabilograms of the test subjects were analyzed according to the area of sway, total locus length, and total locus length per unit area. We found that significant alcohol-induced changes in the sway values in the seated posture were similar to those in the upright posture.
\end{abstract}

Keywords: Body sway; Alcohol load; Seated position; Stabilogram; Electrocardiogram (ECG)

\section{Introduction}

Stabilometry is useful to comprehensively investigate equilibrium function [1]. To increase the diagnostic value of stabilometry, stabilometric procedures and analytical indices of recorded body sway have been established with regard to the total locus length and locus length per unit area [2]. In particular, locus length per unit area is assumed to represent minute variations in postural control and is considered a scale for evaluation of spinal proprioceptive postural control [3]. Body sway is generally measured in a standing position, such as in Romberg's and Mann's postures, and fewer studies on body sway in a seated position have been performed. However, motion sickness that occurs in cars, trains, and ships, visually induced motion sickness that occurs while watching visual contents, and 3-dimensional (3D) sickness, which occurs while watching $3 \mathrm{D}$ video or playing $3 \mathrm{D}$ games, frequently occurs in a seated position. In addition, many jobs are performed in a seated position, such as Video Display Terminal (VDT) operation, office work, and driving, and fatigue accumulates while maintaining a seated position for a prolonged period. Moreover, an upright posture is difficult to maintain for some handicapped and elderly persons. However, there are few methods to objectively evaluate body sway in a seated position.

The mechanism of development of visually induced motion sickness, similarly to motion sickness, is explained with the sensory conflict theory [4], as follows: the equilibrium system receives information input from the visual, vestibular, and somatosensory systems. When the combination of this information concerning body movement is inconsistent with combinations previously established based on experience, spatial localization of self becomes unstable and produces discomfort. Input into the vestibular nuclei located in the brainstem from the visual and somatosensory systems and the cerebellum, in addition to the vestibular system, has been reported [5], suggesting that the nuclei physiologically integrate this sensory information. It is also known that there is a close relationship between the vestibular and autonomic nervous systems anatomically and electrophysiologically [6], which strongly suggests that the equilibrium system is associated with the symptoms of motion sickness, and providing a basis to quantitatively evaluate motion sickness based on body sway, an output of the equilibrium system. Furthermore, when rotation sickness was induced in rats, the histamine level rose in the hypothalamus and brainstem, and this is probably associated with vomiting in motion sickness [7].

There are psychological measurement methods, such as subjective evaluation, and physiological measurement methods, such as measurement related to autonomic nerve activity used to evaluate the influence of visually induced motion sickness on the body. The bestknown psychological method to evaluate visually induced motion sickness is the Simulator Sickness Questionnaire (SSQ) [8]. Evaluation of visually induced motion sickness employing measurement of physiological parameters, such as the heart rate and its variation (RR interval), low frequency of heart rate volubility (LF), high frequency of heart rate volubility (HF), LF/HF, blood pressure, respiratory rate, number of blinks, electrogastrography, skin resistance, and sweat rate has been attempted [9-11]. It has been reported that body sway (total locus length) significantly increased while watching a video in a widestance position (heels were 17 or $30 \mathrm{~cm}$ apart) in a high SSQ score group compared to a low score group [12].

The usefulness of analytical methods of stabilometry is evaluated based on a comparison of healthy subjects and patients with disequilibrium. Experiments involving these subjects are difficult with regard to availability of subjects and reproducibility. One way to overcome these problems is to artificially control disequilibrium and its severity by alcohol ingestion. Many investigators have reported the effects of alcohol on the oculomotor system [13-18] and on the vestibular system through positional nystagmus [19,20]. Postural instability is observed after alcohol ingestion because the cerebellar function controlling the vestibulo-spinal reflex (vestibulo-cerebellar

*Corresponding author: Hiroki Takada, Department of Human \& Artificia Intelligent System, Graduate School of Engineering, University of Fukui, Japan, E-mail: takada@u-fukui.ac.jp

Received February 21, 2012; Accepted April 21, 2012; Published April 25, 2012

Citation: Matsuura Y, Amemori M, Shiomi T, Miyao M, Yoshikawa K, et al. (2012) Evaluation of Body Sway in a Seated Posture after Alcohol Ingestion with an Aim to Evaluate Motion Sickness Caused by Three-Dimensional Images. J Sports Med Doping Stud 2:109. doi:10.4172/2161-0673.1000109

Copyright: (c) 2012 Matsuura Y, et al. This is an open-access article distributed under the terms of the Creative Commons Attribution License, which permits unrestricted use, distribution, and reproduction in any medium, provided the original author and source are credited. 
system) is pharmacologically inhibited by alcohol. The change in the sway of the center of gravity induced by alcohol has been quantitatively evaluated by the correlation between the blood alcohol concentration (BAC) and body sway [17,21]. This instability induced by alcohol affects the mechanism that controls a seated posture. In this study, we verify the hypothesis that changes in the sway of the center of gravity can be detected in the seated position in subjects with high blood alcohol level.

To perform a basic investigation of body sway measurement in a seated position, we conducted an experiment with the artificial control of disequilibrium by alcohol loading and evaluated the sway of the center of gravity in the seated position.

\section{Materials and Methods}

\section{Participants}

The subjects were 7 young males (21-22 years of age) with no past medical history of ear or nervous system disease. The experiment was sufficiently explained to the subjects and written consent was obtained from the subjects. Participants also completed a questionnaire survey before the experiment. Physical information (height, body weight, and sitting height), frequency of alcohol use, and the presence or absence of cigarette smoking was noted.

\section{Materials}

Body sway measurement and electrocardiography were performed. A Wii Balance Board (Nintendo) was used as the stabilometer. The Wii Balance Board shows high temporal resolution, although the spatial resolution is not high compared to existing stabilometers. Existing stabilometers are triangular and large, and not well suited for measurement in a seated position. The coordinates of measurement points in this system are presented in $\mathrm{cm}$, the temporal resolution was $0.01 \mathrm{sec}$, and the sampling frequency was $20 \mathrm{~Hz}$. To investigate the influence of the alcohol load on the cardiovascular system, electrocardiograms were recorded during the experiment using a wireless biological sensor RF-ECG (Medical Electronic Science Institute). The degree of intoxication depends on the cerebral alcohol level, which is difficult to measure. Instead, the breath alcohol level, which is in equilibrium with the cerebral level, was measured using the AT-128 device (Akizuki Denship Tusho).

\section{Design and procedure}

In the experiment, a $1-\mathrm{cm}$ thick plate was placed on a table with a $60-\mathrm{cm}$ height, on which the stabilometer was placed in parallel with the ground. The subject sat up straight with both feet elevated and placed lightly clenched fists on the knees. An electrocardiography electrode was attached to the left chest. After 10 min resting, the sway of the center of gravity was recorded for $1 \mathrm{~min}$ each with open and closed eyes consecutively (seated position stabilometry). In the test with open eyes, the subject gazed at an optotype, a red gaze point that was set at a site $2-\mathrm{m}$ anterior at eye level. The breath alcohol level was measured 2 times immediately before the test with open eyes, and the mean was recorded.

(1) Without alcohol ingestion: Seated position stabilometry was performed, and stabilograms were recorded every $5 \mathrm{~min} 12$ times after the initiation.

(2) With alcohol ingestion: Seated position stabilometry was performed, and stabilograms were recorded every 5 min 12 times after alcohol ingestion.

The order of the above experiments was randomly selected, and the experiments were performed on different days.

\section{Alcohol load}

The volume of alcohol ingestion was set to raise the blood level to $0.6 \mathrm{mmEq}$ following the standard equation of blood alcohol concentration, and the subject drank the undiluted distilled liquor within $30 \mathrm{~s}$. This blood alcohol level is classified as an early phase of slight intoxication, in which the pulse and respiratory rates increase, talk becomes fluent, and behavioral self-restraint decreases [21]. The blood alcohol level, $\mathrm{Cp}$, is determined by the following equation, in which $0.789(\mathrm{~g} / \mathrm{ml})$ is the specific gravity of alcohol, and the numerator and denominator represent the amount (g) and volume of distribution of ingested alcohol, respectively:

$C_{p}[\mathrm{mmEq}]=\frac{(\text { alcohol concentration })[\%] \times(\text { amount of alcohol consumed })[\mathrm{ml}] \times 0.789[\mathrm{q} / \mathrm{ml}]}{0.53[\mathrm{l} / \mathrm{kg}] \times(\text { Weight of subject })[\mathrm{kg}]}$

\section{Data collection}

The $x-y$ coordinates were measured during the sampling time with open or closed eyes, saved as text files, and developed to calculate the parameters. The data obtained with open and closed eyes were converted into a time series of the position of the center of gravity with the rightward and forward directions as positive $\mathrm{x}$ and $\mathrm{y}$ directions, respectively, and the area of sway, locus length per unit area, and total locus length were evaluated. These analytical parameters of stabilometry were employed in previous studies, and we measured them following the definitional equation established by the Japan Society for Equilibrium Research [2].

\section{Data analysis}

The stabilograms of all subjects were recorded at each time point and divided into those with and without alcohol ingestion with open and closed eyes, and the area of sway, locus length per unit area, and total locus length were calculated to investigate differences due to alcohol ingestion.

The paired Wilcoxon signed rank test was employed to evaluate the absence of differences in the population means between the patterns with and without alcohol ingestion as a null hypothesis. In addition, two-way ANOVA comparing the presence or absence of alcohol ingestion and measurement time points as factors was performed to investigate whether the main effects accompanying the alcohol load and maintenance of the posture observed were significant. The significance level, p, was set at 0.05 .

Since inter-individual variation of alcohol metabolism is large, the time course of the heart rate and the breath alcohol level were also investigated.

\section{Results}

Typical stabilograms with and without alcohol ingestion are shown in Figure 1. The sway increased in the locus pattern when alcohol was ingested, compared to that without ingestion.

The mean time course of the area of sway, locus length per unit area, and total locus length are shown in Figure 2-4, respectively. In addition, the mean time course breath alcohol level is shown in Figure 5 . When the eyes were open, the area of sway with alcohol ingestion was about 2 -fold greater than without ingestion, and it was not dependent on the measurement time point. When the eyes were closed, the value measured with alcohol ingestion at 20-35 min was slightly smaller than that without ingestion.

When the eyes were open, the locus length per unit area was shorter when alcohol was ingested compared to that without ingestion. When 
(a)

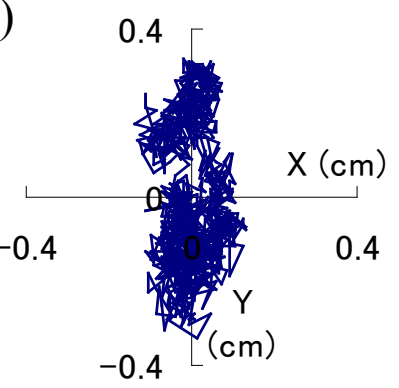

(b)

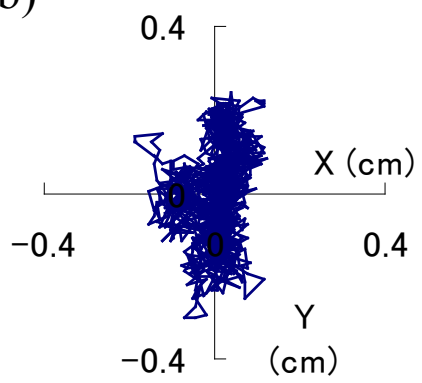

Figure 1: Typical stabilograms observed when subjects viewed the static circle (a), and with alcohol intake after $30 \mathrm{~min}(\mathrm{~b})$.

(a)

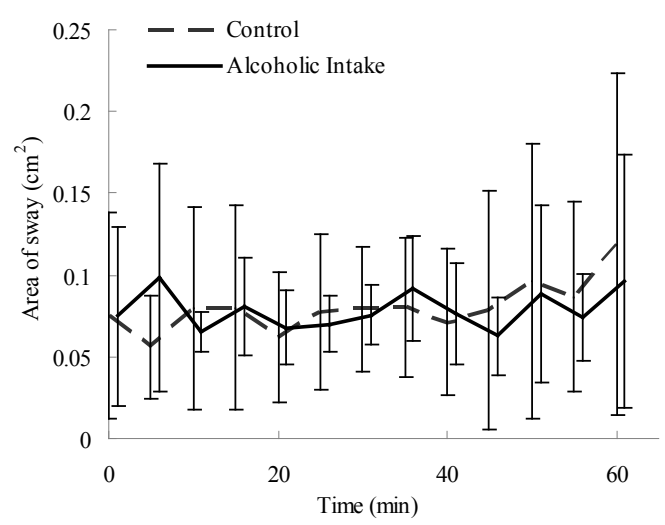

(b)

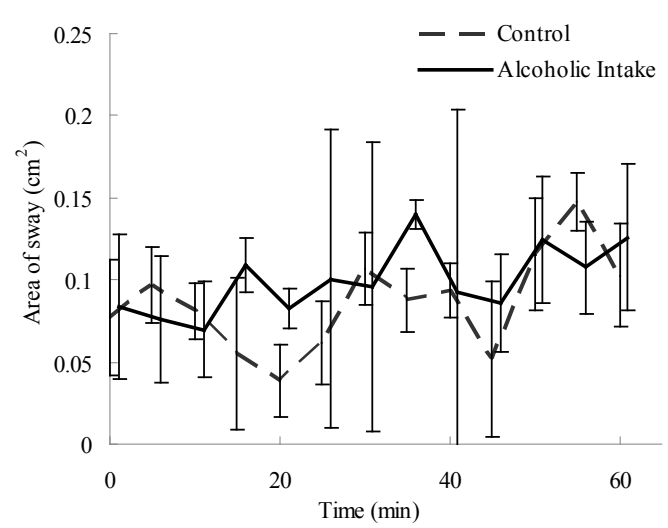

Figure 2: Mean time course of area of sway with alcohol intake vs. control: during open eye tests (a), and during closed eye tests (b).
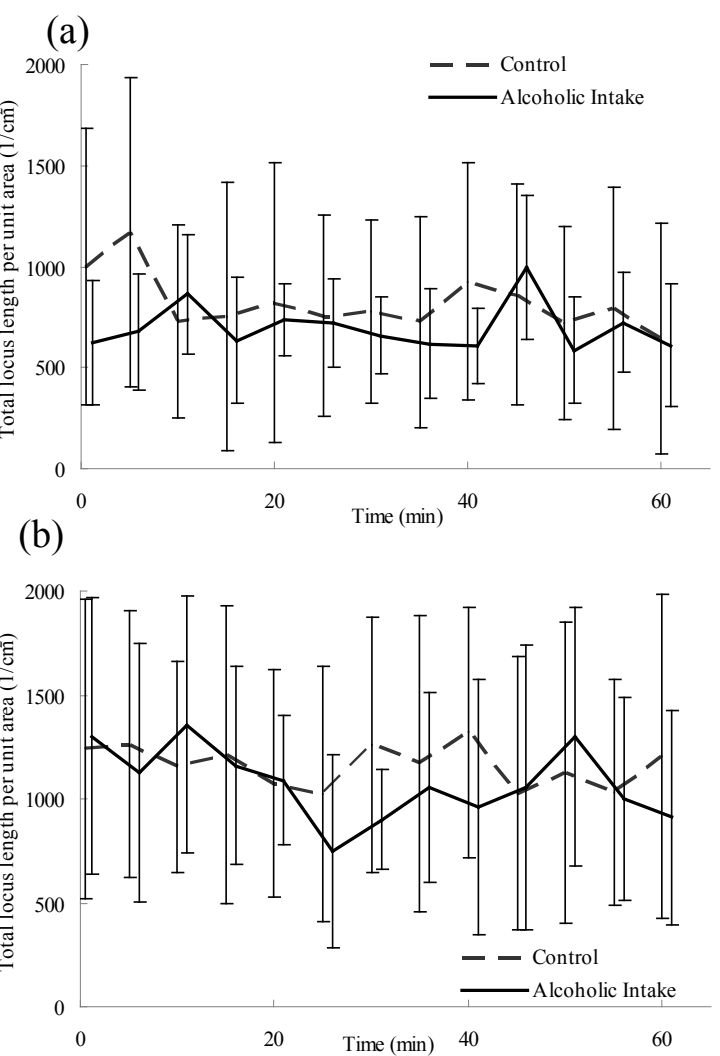

Figure 3: Mean time course of total locus length per unit area with alcohol intake vs. control during open eye tests (a), and during closed eye tests (b).

the eyes were closed, the level at 20-40 min was shorter when alcohol was ingested compared to that without ingestion.

In contrast, no marked difference due to alcohol ingestion was noted in the total locus length when the eyes were open, but the value at 15-30 min was greater when alcohol was ingested vs. without ingestion.

The breath alcohol level rapidly increased for $5 \mathrm{~min}$ from immediately after experiment initiation, followed by a moderate rise until $30 \mathrm{~min}$, and then the level started to decrease at $35 \mathrm{~min}$.

The data from all subjects were divided by open and closed eyes, and the data of each measurement period were analyzed using the Wilcoxon signed rank test. A difference was noted in the population mean of the total locus length between the conditions $(\mathrm{p}<0.05)$ but no difference was noted in the population mean of the area of sway or locus length per unit area between the conditions. A significant alcohol ingestion-induced increase in the total locus length was noted in 3 and 6 analytical periods with open and closed eyes, respectively.

Two-way ANOVA with repeated measures was performed regarding the presence or absence of alcohol ingestion and measurement time point as factors. No interaction was observed among these factors. The main effect of alcohol ingestion on the locus length per unit area was noted when the eyes were open $(\mathrm{p}<0.05)$. A significant effect of alcohol ingestion was noted in the total locus length in tests with both open and closed eyes $(\mathrm{p}<0.01)$.

\section{Discussion}

The analytical parameters of stabilometry in a standing position were used to evaluate sway in a seated position. When the Wii Balance 
(a)

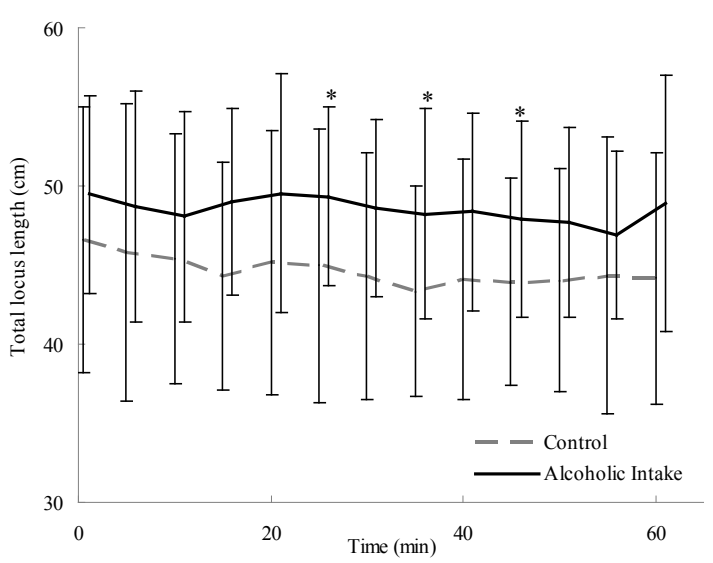

(b)

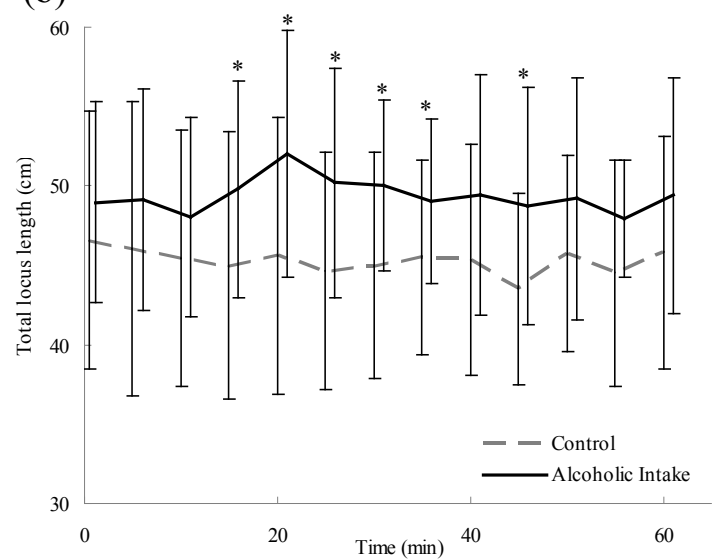

Figure 4: Mean time course of total locus length with alcohol intake vs. control: during open eye tests $(a)$, and closed eye tests $(b)\left({ }^{*} p<0.05\right)$

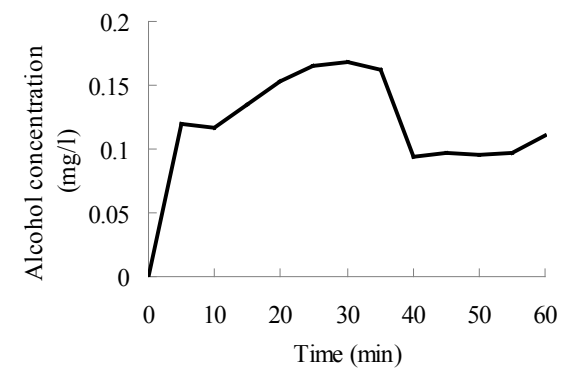

Figure 5: Mean time course of breath alcohol concentration.

Board is used as a stabilometer, the total locus length is useful to detect alcohol ingestion. It also facilitates stabilometry-based non-linear analysis of the human dynamic system to retain and control postures in a seated position [7].

The sway of the center of gravity measured in this study is termed a multicentric pattern [22], showing several minimal points in the time-averaged potential [7] of a mathematical model describing body sway (Appendix). The distance between the minimal points of the time-averaged potential estimated from the stabilogram measured in the seated position tended to increase when alcohol was ingested compared to that without ingestion, and this may have been due to alcohol ingestion-induced changes in the coefficient of the timeaveraged potential of a mathematical model describing the body sway. We have already clarified deformation of the time-averaged potential of a mathematical model describing body sway based on the stabilogram while watching a 3-dimensional film [23].

On analysis of the area of sway and total locus length, the Romberg rate (Romberg rate $=$ values with closed eyes/values with open eyes) was reduced to less than 1 by alcohol ingestion, being judged as a Romberg sign (-). In cerebellar disorders, sway is severe even when the eyes are open [2], and the Romberg rate is less than 1, suggesting that alcohol ingestion does not influence the vestibular function or positional sense of the joints, and that the increase in sway is due to temporary functional inhibition of the cerebellum. This is different from the mechanism of motion sickness caused by watching 3-dimensional video clips. Accordingly, the pattern of structural changes in the timeaveraged potential of the above mathematical model of body sway is different.

The locus length per unit area is viewed as a scale to evaluate the function of spinal proprioceptive postural control [1], representing minute variations in postural control. Regarding this parameter, the main effect of alcohol ingestion was noted on two-way ANOVA $(p<0.05)$ when the eyes were open, indicating that the cerebellar function was temporarily inhibited by alcohol ingestion.

Preceding studies have investigated the influence of alcohol ingestion on body sway in a standing position. It has been statistically demonstrated that it is generally difficult to classify patterns of stabilograms with and without alcohol ingestion employing existing parameters, such as the area of sway, effective area, total locus length, and locus length per unit area in either condition with open or closed eyes [24]. However, differences have been observed between the patterns before alcohol ingestion and at 20-35 min after ingestion in a test with closed eyes [24], supporting the possibility of a different pattern in the sway measured at 15-40 min with closed eyes vs. that without alcohol ingestion in this study. The breath alcohol level exceeded $0.15 \mathrm{mg} / \mathrm{l}$ during these measurement periods (Figure 5), suggesting the presence of a lower limit of intoxication for the detection of alcohol ingestion by seated position stabilometry. Changes in the extent of sway in an upright posture induced by the ingestion of alcohol causing a specific level of drunkenness have been observed. Changes may also be noted in a seated position.

\section{Conclusion}

Stabilometry in a standing position has been established, but measurement of body sway in a seated position has not been established. Thus, sway for which measurement in a seated position is desirable, such as that while watching a film, has to be measured in a standing position, and stabilometry cannot be performed in individuals who cannot maintain a standing position. If body sway measurement in a seated position becomes possible, stabilometry could be performed in these individuals. Furthermore, differences between sway in standing and seated positions could be evaluated, and motion sickness in a simulator could be detected, through which new information could be obtained. In addition, using stabilometry in a seated posture may be significant with regard to public health, as it is possible to assist with the measurement of mental load and fatigue at welfare, health care, and work sites, and broad application and development can be expected. To achieve this potential, an accumulation of basic studies and establishment of a standard evaluation method are necessary. For 
Citation: Matsuura Y, Amemori M, Shiomi T, Miyao M, Yoshikawa K, et al. (2012) Evaluation of Body Sway in a Seated Posture after Alcohol Ingestion with an Aim to Evaluate Motion Sickness Caused by Three-Dimensional Images. J Sports Med Doping Stud 2:109. doi:10.4172/2161-0673.1000109

the next step, it is necessary to further investigate the reliability of a measurement method by increasing the number of subjects.

\section{References}

1. Okawa T, Tokita T, Shibata Y, Ogawa T, Miyata H (1995) Stabilometry: Significance of locus length per unit area (L/A). Equilibrium Research 54: 296

2. Suzuki J, Matsunaga T, Tokumasu K, Taguchi K, Watanabe I (1996) Q\&A and a manual in stabilometry. Equilibrium Research 55: 296-306.

3. Okawa T, Tokita T, Shibata Y, Ogawa T, Miyata H (1996) Stabilometry: Significance of locus length per unit area (1/a) in patients with equilibrium disturbances. Equilibrium Research 55: 283-293.

4. Reason JT, Brand JJ (1975) Motion Sickness, ( $3^{\text {rd }}$ edn), Academic Press England, UK.

5. Barmack NH (2003) Central Vestibular System: Vestibular nuclei and posterior cerebellum. Brain Res Bull 60: 511-541.

6. Balaban CD, Porter JD (1998) Neuroanatomical substrates for vestibuloautonomic interactions. J Vestib Res 8: 7-16.

7. Takeda N, Morita M, Kubo T, Yamatodani A, Watanabe T, et al. (1986) Histaminergic mechanism of motion sickness neurochemical and neuropharmacological studies in rats. Acta Otolaryngol 101: 416-421.

8. Kennedy RS, Lane NE, Berbaum KS, Lilienthal MG (1993) A simulator sickness questionnaire (ssq): a new method for quantifying simulator sickness. Int $J$ Aviat Psychol 3: 203-220

9. Holmes SR, Griffin MJ (2001) Correlation between heart rate and the severity of motion sickness caused by optokinetic stimulation J Psychophysiol 15: 35-42.

10. Himi N, Koga T, Nakamura E, Kobashi M, Yamane M, et al. (2004) Differences in autonomic responses between subjects with and without nausea while watching an irregularly oscillating video. Auton Neurosci 116: 46-53.

11. Yokota Y, Aoki M, Mizuta K, Ito Y, Isu N (2005) Motion sickness susceptibility associated with visually induced postural instability and cardiac autonomic responses in healthy subjects. Acta Otolaryngol 125: 280-285.
12. Scibora LM, Villard S, Bardy B, Stoffregen TA (2007) Wider stance reduce body sway and motion sickness. Proceedings of VIMS2007 18-23.

13. Wilkinson IM, Kime R, Purnell M (1974) Alcohol and human eye movement. Brain 97: 785-792.

14. Umeda Y, Sakata E (1978) Alcohol and the oculomotor system. Ann Oto Rhinol Laryngol 87: 392-398.

15. Barnes GR (1984) The Effects of ethyl alcohol on visual pursuit and suppression of the vestibulo-ocular reflex. Acta Otolaryngol Suppl 406: 161-166.

16. Barnes GR, Crombie JW, Edge A (1985) The effects of ethanol on visualvestibular interaction during active and passive head movements. Aviat Space Environ Med 56: 695-701.

17. Kubo T, Sakata Y, Koshimune A, Sakai S, Ameno K, et al. (1990) Positional nystagmus and body sway after alcohol ingestion. Am J Otolaryngol 11: 416419.

18. Nieschalk M, Ortmann C, West A, Schmäl F, Stoll W, et al (1999) Effects of alcohol on body-sway patterns in human subjects. Int J Legal Med 112: 253260

19. Aschan g, Bergstedt M, Goldberg L, Laurell L (1956) Positional nystagmus in man during and after alcohol intoxication. Q J Stud Alcohol 17: 381-405.

20. Money KE, Myles WS (1974) Heavy water nystagmus and effects of alcohol Nature 247: 404-405

21. Ukai T (1990) Manual of treatment of acute poisoning, the 272 kinds of essentia chemicals and natural poison information 182-183.

22. Fujikake K, Miyao M, Honda R, Omori M, Matsuura Y, et al. (2007) Evaluation of high quality lcds displaying moving pictures, on the basis of the form obtained from statokinesigrams. Forma 22: 199-206.

23. Takada H, Y. Kitaoka Y, Shimizu Y (2001) Mathematical index and model in stabilometry. Forma 16: 17-46.

24. Takada H, Kitaoka Y, Ichikawa M, Miyao M (1996) Physical meaning on geometrical index for stabilometry. Equilibrium Research 55: 283-293. 